\title{
Flexible Performance Prediction of Data Center Networks using Automatically Generated Simulation Models*
}

\author{
Piotr Rygielski \\ Chair of Software Engineering Chair of Software Engineering \\ Samuel Kounev \\ Phuoc Tran-Gia \\ Chair of Communication \\ Networks \\ Institute of Computer Science, University of Würzburg, Am Hubland, 97074 Würzburg, Germany
\{piotr.rygielski@, samuel.kounev@, trangia@informatik.\}uni-wuerzburg.de
}

\begin{abstract}
Using different modeling and simulation approaches for predicting network performance requires extensive experience and involves a number of time consuming manual steps regarding each of the modeling formalisms. In this paper, we propose a generic approach to modeling the performance of data center networks. The approach offers multiple performance models but requires to use only a single modeling language. We propose a two-step modeling methodology, in which a high-level descriptive model of the network is built in the first step, and in the second step model-to-model transformations are used to automatically transform the descriptive model to different network simulation models. We automatically generate three performance models defined at different levels of abstraction to analyze network throughput. By offering multiple simulation models in parallel, we provide flexibility in trading-off between the modeling accuracy and the simulation overhead. We analyze the simulation models by comparing the prediction accuracy with respect to the simulation duration. We observe, that in the investigated scenarios the solution duration of coarser simulation models is up to 300 times shorter, whereas the average prediction accuracy decreases only by 4 percent.
\end{abstract}

\section{Categories and Subject Descriptors}

C.4 [Performance of Systems]: Modeling techniques

\section{Keywords}

performance modeling, data center networks, meta-modeling

\section{INTRODUCTION}

Performance modeling and prediction approaches help system operators to analyze data center performance at system design-time and during operation. Nowadays, data centers

* This work is a part of RELATE project supported by the European Commission (Grant no. 264840ITN). are becoming increasingly big and dynamic due to the common adoption of virtualization technologies. Virtual machines, data, and services can be migrated on demand between physical hosts to optimize resource utilization while enforcing service-level agreements. This makes an accurate and timely performance analysis a challenging problem [17].

In our research, we focus on the network infrastructures of modern virtualized data centers. Network infrastructures in such environments introduce several new challenges for performance analysis. Some examples of such challenges include the growing density of modern virtualized data centers (increasing amount of network end-points), the high volume of intra-data-center traffic (having its source and destination within the same data center), or the new traffic sources introduced in the management layer of virtualized environments (e.g., migration of virtual machines).

Computer networks can be represented in multiple performance modeling formalisms, as for example, domain-specific simulation models, stochastic Petri nets, queueing networks, and stochastic process algebras. The use of a given performance model requires understanding of its formalism and the usual modeling steps. Thus, specific knowledge and experience with the various modeling formalisms are required in order to benefit from their different characteristics. Usually, such knowledge and experience is missing or it is limited to a single modeling formalism.

We propose an approach that requires to model the network using a high-level descriptive language that is generic and contains elements familiar to any network operator. That approach enables the use of multiple different modeling and analysis approaches without requiring in depth expertise in the respective modeling formalisms. Using the high-level descriptive model as a basis, we provide model-tomodel transformations to automatically generate predictive performance models without requiring the operator to have expertise in any of them. For the descriptive modeling of the network infrastructure, we use the Descartes Network Infrastructure (DNI) meta-model as a part of the Descartes Modeling Language (DML) [9]. We refer to meta-models as to modeling languages specified using the MOF formalism (Meta-object Facility) [3]. Network models that are built using the DNI modeling language can be automatically transformed to various predictive models; in this paper we present three predictive models that can be automatically derived: $\mathrm{OMNeT}++$ simulation and two different stochastic models based on Queueing Petri Nets (QPN).

The main contributions of this paper are the following. (i) We propose two new model transformations to automat- 
ically generate performance models based on a single descriptive DNI model instance that is given as an input. (ii) As an intermediate step, we propose a new miniDNI metamodel that is smaller than DNI and offers coarser modeling granularity. Transformation from $D N I$ to miniDNI is performed automatically, so only an instance of the DNI must be provided at the input. (iii) We compare the three predictive models against existing performance models obtained by using the DNI model and the previous transformations partially introduced in $[27,29]$. We reveal the semantic gaps between the model abstractions and the different model solving techniques. (iv) We evaluate the three predictive models with respect to their performance prediction accuracy and the time needed to solve them. Finally, we discuss the results, describe technical challenges, and characterize situations in which a given simulation model performs better than the other models.

The novelty of our approach can be characterized by the following aspects. First, we propose an original descriptive performance modeling language that offers automatic transformation to performance predictive models for performance prediction. Second, thanks to the technology-independence of the DNI modeling language, our approach can be used for modeling novel networking technologies such as network virtualization, and flow-based routing. Third, from a single DNI network model, we automatically provide multiple predictive models that offer different modeling granularity, prediction accuracy, and model solution time. This allows a flexible selection of the prediction model according to the required prediction accuracy and time constrains (e.g., prediction within max. 3 minutes). Fourth, we cover the endto-end performance specifications of the modern data centers by supporting generic server and network virtualization and the link to the deployed software. We do not focus on selected fragments of a specific protocol but model the network as a whole including the deployed software, physical and virtual topology, configuration and traffic.

The rest of this paper is organized as follows. In Section 2, we introduce the foundations, briefly reviewing network performance modeling formalisms and related performance prediction approaches. In Section 3, we introduce our approach to performance modeling and prediction. Section 4 briefly presents the descriptive models and the different model-tomodel transformations. Additionally, we characterize the differences between the generated predictive models. In Section 5, we present our evaluation of the prediction accuracy and the simulation overhead. We describe the model calibration process and formulate technical challenges related to it. Finally, we present our plans for future works and conclude the paper in Section 6.

\section{FOUNDATIONS AND RELATED WORK}

There is a large body of existing work on performance modeling of communication networks. The related work can be divided into two main areas: predictive models for performance analysis and approaches that rely on the model-based generation of predictive models.

\subsection{Performance Models for Data Center Net- works}

There exist many performance modeling formalisms suitable for modeling networks, for example [16, 25]. Existing modeling approaches are mostly based on stochastic models such as classical product-form queueing networks, layered queueing networks, stochastic Petri nets, stochastic simulation models, and so on. We can distinguish domain-specific performance models (e.g., network simulators) and general purpose models that can be used to model the networks.

The most popular network simulation frameworks are OM$\mathrm{NeT}++[31]$ and ns-2/3 [26] — both discrete event simulators. In [26], $n s-2$ has been claimed for many years to be standard simulator for academic network research. Modeling using $n s$ - 2 requires extensive knowledge of programming in $\mathrm{C}++$ and thus required time investment to learn how to model. Similar observations apply to OMNeT ++ -almost all protocol-specific models need to be implemented by hand. According to [34], further similar simulators include: open$W N S$, OPNET, GTNetS, and IKR simulation library. All these simulators focus on medium-detailed modeling of the popular TCP/IP protocol stacks; extensions to support further popular protocols are often available as well. There exist also simulators that are tightly bound to a given protocol or even to a concrete implementation of that protocol. An example is the Venus simulator [12]; the authors use the TCP implementation extracted from FreeBSD v9 kernel to keep the simulation accuracy maximized.

On the other hand, there exist general-purpose performance modeling formalisms that can also be applied for network modeling. To such formalisms we account, for example, queueing networks, layered queueing networks, stochastic Petri nets, stochastic process algebras, Markov chains, analytical estimation methods (bounds analysis). In [25], Puigjaner reviewed selected general-purpose performance modeling formalisms and their applicability in the communication networks area.

In this paper, we use, among others, stochastic Petri nets as a predictive model. Regarding the stochastic Petri nets, such as queueing Petri nets, and their usage in a networking context, so far they have mainly been used to model specific network components at a high level of detail but with limited scope as, for example in [37, 21]. However, an end-to-end modeling approach is crucial in modern virtualized systems. The authors of [33] claim: „The fundamental problem is that the simple textbook end-to-end delay model composed of network transmission delay, propagation delay, and router queueing delay is no longer sufficient. Our results show that in the virtualized data center, the delay caused by end host virtualization can be much larger than the other delay factors and cannot be overlooked."

Domain-specific simulators cover in high detail selected performance-relevant aspects of communication networks. To the other extreme - regarding the level of abstraction of the models - we account black-box approaches, where the performance metrics of interest are calculated using simple mathematical equations based on a few general network parameters. The black-box modeling approach is normally applied in cases where the networks are not in the main focus of the analysis, but their influence on performance cannot be neglected. Such approaches can be found in [14, 20].

\subsection{Approaches based on Model-to-model Transformations}

The wide variety of performance models makes it challenging to select the proper models and learn them extensively to model the performance precisely. Model-based approaches assume that there exists a single descriptive model 


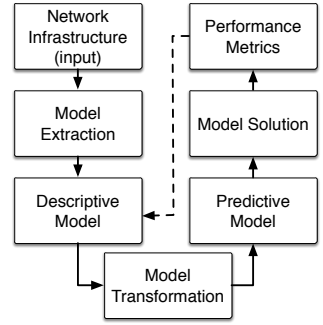

(a)

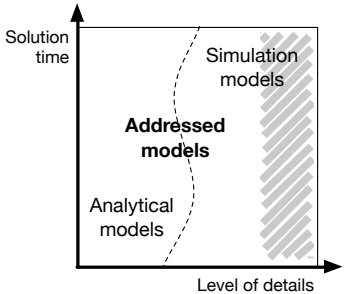

(b)
Figure 1: (a) Process of performance prediction based on model-to-model transformations. (b) Abstract division of prediction models into analytical and simulation models. Models with very fine modeling granularity (gray area) are not our target.

and all predictive models are derived automatically using model transformations. The general performance prediction process based on model-to-model transformations is presented in Figure 1a. A model of a real network is built and stored in a descriptive model with performance annotations, whereas the rest of the process is automated and can deliver as many different predictive performance models as many model transformations are available. Some authors, e.g., [23], use the prediction results to further refine their models (dashed line in Fig. 1a).

Approaches based on model-to-model transformations stem from software engineering community. UML models are used to analyze various software-related metrics [5]. Software architecture models are annotated with performancerelevant information and later transformed into predictive models (e.g., [7, 36]). Software architecture models have been further extended to also include information about the hardware resources and the deployment of software components. Among the hardware-related information also very simple network models have been considered. Acknowledging the wide variety of such infrastructure models (e.g., [6, $10]$ ), we further review the models that include performance of the network infrastructures.

End-to-end performance analysis requires taking into account multiple performance-influencing factors such as computing resources, deployment middleware, storage, and networks. Networks are usually abstracted in such complex models and represented as black-box statistical models. In [6], the authors model the network as a linking resource represented as a black-box analytical function, abstracting the network configuration, topology and traffic patterns. On the other hand, the authors in $[11,13,18]$ propose highlydetailed protocol-level simulation models that cover only selected parts of network infrastructure. Other approaches model the network environment, however without providing support for performance analysis, e.g., [1, 24, 8].

The black-box performance modeling approaches do not consider the internal network structure and topology while the highly-detailed, protocol-level models focus only on selected parts of the network infrastructure and do not capture the link to the running applications and services that generate the traffic. We address these issues by providing a generic modeling formalism that can be transformed to multiple models with different modeling granularities, whereas in each of those models we include the whole picture of the network in contrast to concentrating on a selected fragment.

\section{APPROACH}

We divide the area of performance models as shown in Figure 1b. We intentionally exclude the simulation models with high level of details (striped area in Fig. 1b) because they require specifying too many low level input parameters and usually do not model the infrastructure in an end-toend manner. However, we do not question usability of those models and their high prediction accuracy; we recommend using these models when maximum modeling accuracy is a major requirement.

Using our model-based approach, we address the medium and low-detailed predictive models (the non-stripped area in Fig. 1b) that can be generated automatically using model transformations. Among the addressed predictive models, we consider models with various level of detail (respectively prediction accuracy) and with different model solution time. Using automatic model generation methods enables us to pick the proper model according to the given situation: quick but less detailed solution or longer simulation providing more accurate prediction. We call this flexible performance prediction because by modeling the infrastructure once, one can freely pick the suitable predictive model or even use multiple models in parallel.

The modeling approach we propose is based on a metamodel for modeling network infrastructures in virtualized data centers. This meta-model, which we refer to as Descartes Network Infrastructure (DNI) meta-model, is part of our broader work in the context of the Descartes Modeling Language (DML) [9], an architecture-level modeling language for modeling Quality-of-Service and resource management related aspects of modern dynamic IT systems, infrastructures and services. The DNI meta-model has been designed to support describing the most relevant performance influencing factors that occur in practice while abstracting finegranular low level protocol details.

In our approach, instances of the DNI meta-model are automatically transformed to predictive stochastic models (e.g., stochastic simulation models) by means of model-tomodel transformations. The approach supports the implementation of different transformations of the descriptive DNI models to underlying predictive stochastic models (by abstracting environment-specific details, transformations to multiple predictive models are possible), thereby providing flexibility in trading-off between the overhead and accuracy of the analysis. In Section 4, we present the models, model transformations and the resulting predictive models.

\section{MODELS AND TRANSFORMATIONS}

\subsection{Meta-models}

\subsubsection{DNI}

We use the DNI meta-model [29] to describe a network infrastructure. The DNI meta-model (initially presented in the work-in-progress paper [30] with technical details presented online in the manual [28]) is intended to describe the common network components in an abstract manner. To such common network components we account, for example: network nodes (physical and virtual), links (physical 
and virtual), traffic sources, flows, and routes. DNI captures the most important performance-relevant properties of a network in a generic manner. It allows one to describe any network (not only packet-switched) and is not bound to any particular network technology.

The DNI meta-model covers three main parts of every data center network infrastructure: structure, traffic and configuration. The first part of the DNI meta-model - network structure - is intended to model the topology of the network. The meta-model contains entities such as nodes and links connected through network interfaces. All nodes, links and interfaces can either be physical or virtual; each virtual network element is hosted on a physical node. We describe the performance-relevant parameters of every element in the model. We distinguish end nodes (e.g., virtual machine, server) and intermediate nodes (e.g., switch, router), because their performance descriptions are different.

In the DNI meta-model, network traffic is generated by traffic sources that are deployed on end nodes. Each traffic source generates traffic flows that have exactly one source and possibly multiple destinations. The flow destinations are located in nodes and can be uniquely identified by a set of protocol-level addresses. Flows can be composed in a workload model that defines how each flow is generated (e.g., with sequences, loops, or branches). In this paper, we describe a flow by specifying the amount of transferred data. The meta-model and its transformations can be systematically extended to support other flow descriptions, e.g., [15].

The configuration of a network contains information about routes, protocols and protocols stacks. We use this information to calculate the paths in the topology graph and to coarsely estimate the overheads introduced by the protocols. In the model, we describe a snapshot of the current routes in the system, disregarding if the system uses static or dynamic routing. The protocols are described by a set of generic parameters (i.e., such parameters, that can be applied to any protocol) such as, for example, overheads introduced by the data unit headers.

In the meta-model, a route consists of a list of references to network interfaces. The routing can be described in two ways: the classical with routes defined between the pairs of nodes (source-destination), and the flow-based description with a route defined for every flow individually. Even if there are multiple flows deployed on the same node and each having the same destination address, the routes can be calculated individually for each of them in contrast to the classical routing representation, where all flows would follow the same route. The flow-based routing representation enables the modeling of the software-defined networks, for example, based on the OpenFlow protocol [22]. We provide a model transformation to convert between the classical and the flow-based routing description (depicted as „Routing format conversion" in Fig. 3). We briefly describe the transformation in Section 4.2.

\subsection{2 miniDNI}

Despite the high level of abstraction of DNI, it still requires many parameters to be provided as input. To reduce the amount of input data, we provide a smaller version of the DNI meta-model; we call it miniDNI. The entities included in the miniDNI are depicted in Figure 2.

In the miniDNI meta-model, we abstract the following information. First, we abandon the virtual entities (links

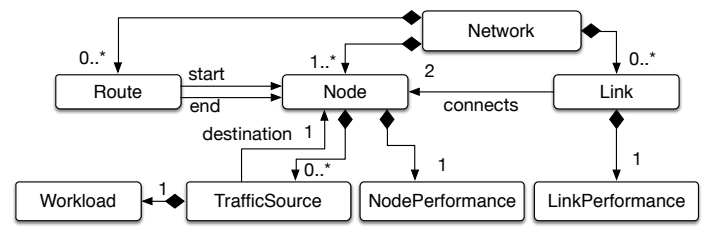

Figure 2: miniDNI meta-model

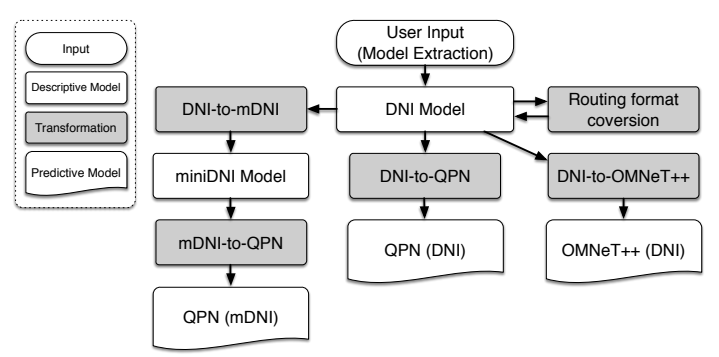

Figure 3: Models and model transformations

and nodes) and provide only one generic representation of them. Second, we remove the NetworkInterfaces; the information included in a NetworkInterface is now merged into the Link. Third, we simplify the descriptions of the traffic sources and workflows. In miniDNI, a workflow specifies only a size of a message and the number of messages per second (for brevity, not shown in Fig. 2. For more details, see [28]) without defining what a message actually is. Finally, we abstract out all information about protocols used in the network. From the DNI's NetworkConfiguration, we keep only simplified information about routes in the network. The routes are flow-based, which means that there is at least one route defined for every traffic source.

\subsection{Transformations}

The meta-models presented in Section 4.1 are transformed into predictive models using model transformations. We use the Epsilon languages [19] for model transformations. The models and transformations between them are presented in Figure 3.

Initial ideas and sketches of the transformations DNI-to$Q P N$ and DNI-to-OMNeT++ were presented in the workshop papers [29, 27]. Since then, the transformations have been finalized and extended. The transformations generate models of OMNeT++ version 4.5 with the INET library Version 2.4 and SimQPN version 2.1 respectively. The technical details of the DNI-to-QPN and DNI-to-OMNeT++ transformations are available in the manual [28], the referred workshop papers and in the source code that is available under http://go.uni-wuerzburg.de/aux. Due to limited space, we describe in this section the three new transformations: DNI-to-mDNI, $m D N I$-to- $Q P N$, and the routing format conversion; for technical details, please refer to [28].

The first transformation-DNI-to-mDNI - transforms DNI models into respective miniDNI models. In the transformation, some information is lost because the miniDNI model contains less details than the respective DNI model. We provide an overview of transformation rules in Table 1.

The obtained miniDNI model is a descriptive model and needs to be further transformed in order to deliver perfor- 
Table 1: Selected transformation rules from the DNI-to-mDNI transformation

\begin{tabular}{|l|l|l|}
\hline DNI & miniDNI & Comments \\
\hline${ }^{*}$ Node & Node & Information about the physical and virtual nature of a Node is abstracted. \\
\hline${ }^{*}$ Link, ${ }^{*}$ NetworkInterface & Link & $\begin{array}{l}\text { Information about the physical and virtual nature of a Link is abstracted. A Link connecting two } \\
\text { NetworkInterfaces is transformed into a Link connecting two miniDNI Nodes. }\end{array}$ \\
\hline $\begin{array}{l}\text { SoftwareComponent, } \\
\text { TrafficSource }\end{array}$ & TrafficSource & Information about the software is abstracted. TrafficSource is the entity that represents traffic generation. \\
\hline $\begin{array}{l}\text { Flow, }{ }^{*} \text { Action, } \\
\text { WorkflowDescription }\end{array}$ & Workflow & $\begin{array}{l}\text { Information about traffic generated by a traffic source is aggregated into parameters: messageSize and } \\
\text { numberOfMessagesPerSecond. }\end{array}$ \\
\hline NetworkConfiguration & Route & The network configuration is abstracted (except of routes). \\
\hline
\end{tabular}

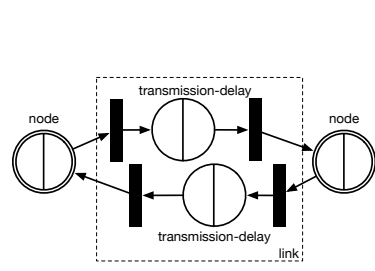

(a)

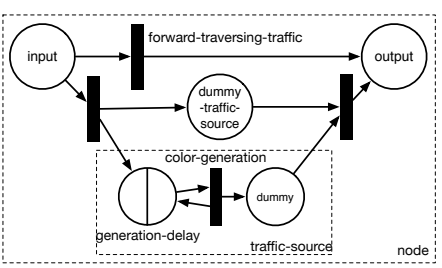

(b)
Figure 4: QPN representation of (a) Link, (b) Node, and TrafficSource in the $m D N I-t o-Q P N$ transformation.

mance predictions. We present the $m D N I-t o-Q P N$ transformation that transforms a miniDNI model into a Queueing Petri Net (QPN) model. The QPN model resulting from the $m D N I-t o-Q P N$ transformation differs from the model obtained in the DNI-to-QPN transformation. Both QPN models represent the same network but they differ in the amount of details being modeled. We briefly present the $m D N I-t o-Q P N$ transformation.

The miniDNI model describes the structure of a network using Nodes and Links. These two entities are mainly used to generate the structure of the respective QPN. Every Node is represented as a subnet. Connections between subnets are obtained by transforming Links into pairs of queueing places connected to Subnets using immediate transitions.

The QPN representation of a Link, presented in Figure 4a, consists two queueing places where contention effects from the network interfaces happen. The delays in transmissiondelay places are calculated using information included in the LinkPerformance entities. Two pairs of immediate transitions are required by the QPN formalism to connect two consecutive places. The transitions include modes - one for each token color traversing the link. The colored tokens represent traffic in the QPN. There is exactly one color for each TrafficSource. Colors are assigned to places and transitions based on the information contained in the Route entities. The transformation reads the routing information and assigns a color to the place or transition if the respective link or node carries the traffic of the given flow.

The colored tokens representing network traffic are generated in the Nodes. The structure of the QPN representing a Node is presented in Figure $4 \mathrm{~b}$ and consist of three parts. The first part is the forward-traversing-traffic transition. It is responsible for processing the tokens from the input to the output place if the Node is neither the source nor the destination of the traffic represented by the token color. The transition is removed from the QPN model if a given node is not a traversal node for any color. The information about traversal nodes is derived from the Route entities. The second part consist of the ordinary place called dummy-traffic-source. This place is necessary to keep the QPN graph connected in case the respective node does not act as traffic generator, nor destination or traversal node. The third part of the subnet contains the set of traffic sources responsible for generating tokens representing traffic. Each traffic source (see dashed frame in Figure 4b) generates tokens of one color. A single token represents a single message of the given size. The intergeneration time - derived from Workload entityis modeled as a parameter of the generation-delay places. The ordinary place dummy acts as a link between the two neighboring transitions, as QPN does not allow to connect two transitions directly.

The transition between the input place and the traffic source is responsible for removing incoming tokens - it contains modes that remove every token that arrives to it. By such representation, we model the traffic as an open workflow. Only tokens having destination in the given node are removed; other (traversing) tokens are passed to the output place using the forward-traversing-traffic transition.

Each QPN's representation of the miniDNI node supports additionally special token color called ether that was introduced to guarantee the Petri net graph to be connected and thus fulfill the liveness property. Further details of the transformation and of the generated QPN model (e.g., token colors, the transition modes, the incidence functions) is available in the manual [28] or can be obtained by looking at the source code that is available online under http: //go.uni-wuerzburg.de/aux.

The third transformation converts the format of the routing representation between flow-based and classical description (and vice versa). Transforming classical routing to the flow-based format is trivial; for each flow a route is built that contains the ordered list of intermediate nodes. Reverse transformation works analogously.

\subsection{Semantic Gaps}

In the presented approach, we automatically generate three predictive models out of a single descriptive model. In this section, we compare the predictive models by describing which predictive model supports which performance-relevant network features. A selection of features and metrics of the predictive models is presented in Table 2. We briefly discuss the main differences and then evaluate them in Section 5.

OMNeT++ along with the INET framework supports most popular network protocols, including IP, TCP, and UDP. Moreover, it generates network traffic on the packet level and therefore it allows complex traffic pattern generations (e.g., as specified in [32]). The implementation of QoS (Quality of Service) queue schedulers is also possible, however only sim-

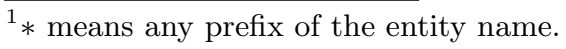


Table 2: Selected features of the compared predictive models

\begin{tabular}{|l|c|c|c|}
\hline Feature & $\begin{array}{c}\text { OMNeT } \\
\text { DNI }\end{array}$ & $\begin{array}{c}\text { QPN } \\
\text { DNI }\end{array}$ & $\begin{array}{c}\text { QPN } \\
\text { mDNI }\end{array}$ \\
\hline Intermediate-/end-nodes & + & + & - \\
\hline Physical/virtual nodes & + & $\oplus$ & - \\
\hline Traffic patterns & + & + & - \\
\hline Packet-level traffic generation & + & - & - \\
\hline QoS schedulers & $\oplus$ & $\odot$ & - \\
\hline TCP/IP Protocol & + & $\odot$ & - \\
\hline UDP Protocol & + & $\oplus$ & - \\
\hline \multicolumn{4}{|c|}{ Performance metrics } \\
\hline Throughput time series & + & - & - \\
\hline Throughput distribution & + & $\oplus$ & $\oplus$ \\
\hline End-to-End delay time series & $\odot$ & - & - \\
\hline End-to-End delay distribution & $\odot$ & $\odot$ & $\odot$ \\
\hline + support, $\oplus$ partial support, $\odot$ extension possible,,- no support \\
\hline
\end{tabular}

ple scheduling algorithms are provided in the INET library; more complex algorithms must be programmed manually.

In contrast to OMNeT++, the QPN model can mimic selected features of the UDP protocol behavior (e.g., dropping excessive traffic, unreliable transmission). Also coarse behavior of the TCP protocol can theoretically be modeled using QPNs, however, we do not provide such implementation in this paper. Although detailed traffic patterns are supported, the packet-level traffic generation is not modeled in the QPN model. A single unit of traffic is a message of a given size (that in reality represents a, so called, packet train or flow) and the traffic patterns can be modeled at the granularity of messages. In the QPN model, we support distinction between end- and intermediate nodes (see [27]), however, every virtual node is modeled as a VM hosted on a physical node (independent of its nature: switch or server).

The most coarse-grained QPN model-obtained in the $m D N I-t o-Q P N$ transformation-abstracts most of the considered features. Nodes are modeled in the same manner without distinguishing their roles: virtual, physical, endor intermediate nodes; the differences are encoded in the numeric values of parameters describing their performance whereas the structure of the QPN remains the same. The model of the traffic is flat and is modeled at the level of messages; only message size and average numbers of messages per unit of time are modeled. Information about protocols, their behavior, and overheads are abstracted.

All performance models analyze throughput as the main performance metric, however, only $\mathrm{OMNeT}++$ provides detailed throughput values for each moment of the simulation; the other models provide aggregated statistics. Despite the different granularity of traffic modeling (packet-level in $\mathrm{OMNeT}++$ versus message-level in QPNs), analysis of the end-to-end transmission delay is possible in both formalisms. The necessary extensions can be easily added, however, the implementation is still considered as a future work.

\section{EVALUATION}

In this section, we evaluate the predictive models presented in Section 4, considering their prediction accuracy and the model solution time (i.e., simulation duration).

\subsection{Case Study}

The system under study is a traffic monitoring application based on results from the Transport Information Monitoring Environment (TIME-EACM) project [4] at the Uni-

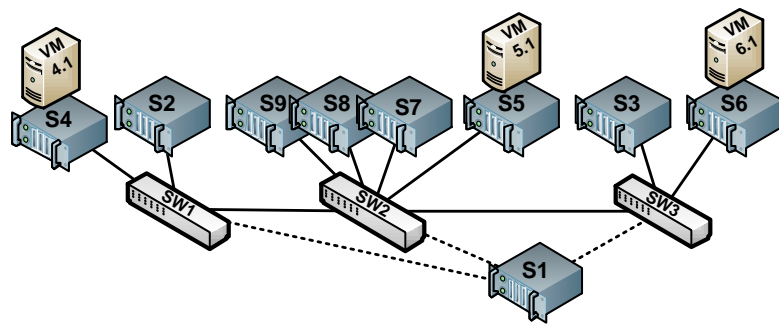

Figure 5: Network topology used in the experiment. Dashed links are used for monitoring, solid links for data traffic. Server $S 1$ is the experiment controller.

versity of Cambridge. The system consists of multiple distributed components and is based on the SBUS/PIRATES (short SBUS) middleware.

In the case study, we consider two types of SBUS components: camera components and license plate recognition (LPR) components. The cameras are located in the city and take pictures of cars that are speeding or entering a paid zone. Each camera is connected to a respective SBUS component that sends the picture together with a time stamp to an LPR component. The LPR components are deployed in a data center due to their high consumption of computing resources. LPRs receive the pictures emitted by cameras and run a recognition algorithm to identify the license plate numbers of the observed vehicles.

\subsection{Reference Models}

Reference models serve as a baseline to evaluate the prediction accuracies of the generated predictive models. We consider two reference models in the evaluation. First, we consider the original SBUS implementation from the TIMEEACM project. Second, we consider the uperf benchmark [2] configured to mimic the traffic generated by the SBUS implementation and to abstract the application logic of the SBUS components (Camera, LPR) used in the case study. In [29], uperf was shown to correctly represent the traffic patterns of the SBUS implementation allowing the system to scale to picture generation frequencies that cannot be handled by the original SBUS implementation.

\subsection{Hardware and Configuration}

The system under study was deployed in a local data center consisting of nine servers and three switches. Each server is equipped with four $1 G b p s$ Ethernet ports. The switches are HP ProCurve 3500yl. The physical topology and the configuration of the network environment is depicted in Figure 5. Server $S 1$ is used to control the experiment and to acquire the monitoring data from switches using SNMP. Two servers, $S 2$ and $S 3$, are native (not virtualized). The nodes $S 4-S 6$ are hosting VMs, whereas $S 8$ hosts VMware Control Center and $S 9$ is a storage.

\subsection{Model Calibration}

The DNI model describes the presented network, however, to be useful for performance prediction, it first needs to be annotated with performance-relevant information.

\subsubsection{Network Structure}

Annotating the network structure-mainly the processing rates of the interfaces - is done straightforward by us- 
ing the values provided in the technical specifications of the respective devices. In such way, we describe all network interfaces of switches and physical hosts. The links themselves are physical media and the only factor that influences their performance is the propagation delay, which is specified as a tabulated value for the underlying network. Also the switching delays are specified in the technical documentation. Much more challenging is to calibrate other parameters, like for example, speeds of virtual entities, protocol overheads, and traffic models.

\subsubsection{Protocols}

Information about protocol overheads is extracted from the operating system. Parameters like the MTU (Maximum Transmission Unit) and the length of a data unit header play an important role for calculating protocol overheads: every message is divided by the MTU value (to estimate the number of packets) and then the header overhead is added to each packet. The size of the message is then increased by the sum of packet overheads. For DNI-to-QPN, this calculation is done in the transformation; $\mathrm{OMNeT}++$ simulates it internally, whereas $m D N I-t o-Q P N$ ignores the protocol overheads.

\subsubsection{Traffic Patterns}

The traffic patterns are modeled manually on the protocol level based on traffic monitoring tools or tcpdump traces. Silence intervals between sending consecutive messages are calculated manually and averaged over the duration of the entire experiment. In case of any parameter variability, a probability distribution can be constructed. Additionally, we verify if the intergeneration times configured in the software (e.g., ,send a picture every $10 \mathrm{~ms}$ ") match the times that are derived from the tcpdump. For our experiments, we describe this process, its consequences, and challenges in section 5.6.

\subsubsection{Server Virtualization}

Another challenging part is the extraction of the parameters that describe the virtual entities. In case of data transmission from one VM to another collocated VM, the data is in fact copied between memory cells. The exact path of the data and the overheads depend on the hypervisor type and the delays are difficult to estimate a priori. Additionally, the performance of the network bridge that is built-in in the hypervisor depends on the system load, which is not reflected in DNI since all model input parameters (e.g., resource demands) are modeled as load-independent.

The values of the performance parameters describing virtual entities are finally estimated experimentally and modeled in a black-box manner. In this case study, we examine the performance of the hypervisor-emulated network by running stress tests defined in uperf. The average bandwidth achieved in the tests is used directly to describe the speeds of the virtual network interfaces in VMs and in the hypervisor bridge.

\subsection{Measurements}

To obtain the baseline performance values, we measure the amount of the traffic flowing through the real network interfaces of all switches. We use the counters located in the switches to measure the number of bytes transmitted through each interface. We read the values of the coun- ters through SNMP every second and calculate the average throughput for that interval. Server $S 1$ makes measurements using an isolated VLAN. The experimental network was isolated from other networks (e.g., the Internet). During the measurements, all intergeneration times were modeled as exponentially distributed; confidence intervals are calculated for a significance level of $\alpha=0.05$. In every experiment, we send predefined amount of pictures (5 000 for each camera) and execute the experiment 30 times for SBUS, uperf, and $\mathrm{OMNeT}++$ and once for QPN (SimQPN cares internally about the required amount of repetitions).

\subsection{Results}

We evaluate the proposed approach in two scenarios. The goal of scenario 1 is to evaluate the accuracy of throughput prediction. We deploy the camera components and the LPR components and configure the communication between the components according to the following plan: $\mathrm{S} 2 \rightarrow \mathrm{VM} 4.1$, $\mathrm{S} 2 \rightarrow \mathrm{VM} 5.1, \mathrm{~S} 3 \rightarrow \mathrm{VM} 6.1$, and $\mathrm{S} 3 \rightarrow \mathrm{VM} 5.1$. In this scenario, we increase the amount of transmitted pictures per second by decreasing the think time between sending consecutive pictures to: 100, 50, 35, 20, and 10ms respectively. We also measure the wall-clock times for every simulation and for the SBUS experiment. In scenario 2, we compare simulation durations for growing traffic intensity and the increasing number of servers in the data center.

\subsubsection{Prediction Accuracy}

In the evaluation of the prediction accuracy, we measure the throughput on the switch ports (for the reference models: SBUS and uperf) and compare them against the values predicted by the generated simulation models. In this experiment, we measure throughputs on selected switch interfaces: $\mathrm{S} 2 \rightarrow \mathrm{SW} 1, \mathrm{~S} 3 \rightarrow \mathrm{SW} 3$, and $\mathrm{SW} 2 \rightarrow \mathrm{S} 5$. In scenario 1, we expect the monitored throughputs to be equal on each interface. The variations may happen when the network capacity is saturated as the TCP protocol may divide the throughput unequally among the flows. The results are presented in Table 3.

Based on the gathered results, we observe the expected equalities in the measured and predicted throughputs for scenarios 1A-1D. By unsaturated network, the two reference models performed similarly (max. 5.3\% throughput variation relatively). For scenario $1 \mathrm{E}$ (saturated network), we observe wide confidence interval for the SBUS model. This phenomenon was observed in our previous workshop work [29] and is caused by software performance bottlenecks in the original SBUS implementation. For that scenario, we use uperf as the reference model. The predictions of the generated models follow the measured trends with average relative error of $7.4 \%$ for OMNeT++, $9.9 \%$ for the QPN(DNI), and $11.4 \%$ for the QPN(miniDNI).

OMNeT++. The OMNeT++ model predicted the throughput with the lowest average relative prediction error $7.4 \%$ (calculated as the relative difference between the mid points of confidence intervals). However, in scenario 1D, OMNeT++ reported the highest inaccuracy of $19 \%$ mispredicting the throughput maximally by $117 \mathrm{Mbps}$ for the link SW2 $\rightarrow$ S5. We have investigated the inaccuracy of the model and formulate the following observations and challenges.

The first challenge is the TCP protocol configuration. From the generated simulators only $\mathrm{OMNeT}++$ is able to mimic the behavior of the TCP protocol. TCP relies on multi- 
Table 3: Scenario 1A-E: measured and predicted throughput in mega-bits per second

\begin{tabular}{|c|c|c|c|c|c|c|c|c|}
\hline \multirow[t]{2}{*}{ Link } & \multicolumn{2}{|c|}{$\begin{array}{c}\text { SBUS } \\
\text { reference1 }\end{array}$} & \multicolumn{2}{|c|}{$\begin{array}{c}\text { uperf } \\
\text { reference } 2\end{array}$} & \multicolumn{2}{|c|}{$\begin{array}{c}\text { OMNeT } \\
\text { DNI }\end{array}$} & \multirow{2}{*}{$\begin{array}{l}\text { QPN } \\
\text { DNI } \\
\text { avg. }\end{array}$} & \multirow{2}{*}{$\begin{array}{l}\text { QPN } \\
\text { mDNI } \\
\text { avg. }\end{array}$} \\
\hline & ICI & $\mathrm{uCI}$ & ICI & $\mathrm{uCI}$ & ICI & $\mathrm{uCI}$ & & \\
\hline \multicolumn{9}{|c|}{ Scenario 1A (think time $100 \mathrm{~ms}$ ) } \\
\hline $\mathrm{S} 2 \rightarrow \mathrm{SW} 1$ & 205 & 216 & 211 & 219 & 199 & 224 & 202 & 202 \\
\hline $\mathrm{SW} 2 \rightarrow \mathrm{S} 5$ & 205 & 215 & 211 & 219 & 199 & 214 & 202 & 202 \\
\hline $\mathrm{S} 3 \rightarrow \mathrm{SW} 3$ & 204 & 215 & 210 & 220 & 200 & 223 & 202 & 202 \\
\hline \multicolumn{9}{|c|}{ Scenario 1B (think time $50 \mathrm{~ms}$ ) } \\
\hline $\mathrm{S} 2 \rightarrow \mathrm{SW} 1$ & 430 & 449 & 385 & 410 & 407 & 448 & 450 & 450 \\
\hline $\mathrm{SW} 2 \rightarrow \mathrm{S} 5$ & 431 & 447 & 390 & 403 & 404 & 437 & 450 & 450 \\
\hline $\mathrm{S} 3 \rightarrow \mathrm{SW} 3$ & 430 & 447 & 383 & 410 & 408 & 442 & 450 & 450 \\
\hline \multicolumn{9}{|c|}{ Scenario $1 \mathrm{C}$ (think time $35 \mathrm{~ms}$ ) } \\
\hline $\mathrm{S} 2 \rightarrow \mathrm{SW} 1$ & 541 & 562 & 496 & 539 & 471 & 530 & 578 & 578 \\
\hline SW2 $\rightarrow$ S5 & 526 & 548 & 505 & 528 & 454 & 517 & 578 & 578 \\
\hline S3 $\rightarrow$ SW3 & 524 & 551 & 495 & 538 & 419 & 484 & 578 & 578 \\
\hline \multicolumn{9}{|c|}{ Scenario 1D (think time $20 \mathrm{~ms}$ ) } \\
\hline $\mathrm{S} 2 \rightarrow \mathrm{SW} 1$ & 631 & 640 & 579 & 652 & 702 & 764 & 675 & 675 \\
\hline $\mathrm{SW} 2 \rightarrow \mathrm{S} 5$ & 639 & 648 & 583 & 640 & 689 & 752 & 675 & 675 \\
\hline $\mathrm{S} 3 \rightarrow \mathrm{SW} 3$ & 416 & 426 & 575 & 648 & 657 & 728 & 675 & 675 \\
\hline \multicolumn{9}{|c|}{ Scenario $1 \mathrm{E}$ (think time $10 \mathrm{~ms}$ ) } \\
\hline $\mathrm{S} 2 \rightarrow \mathrm{SW} 1$ & 686 & 941 & 882 & 941 & 914 & 942 & 978 & 1074 \\
\hline $\mathrm{SW} 2 \rightarrow \mathrm{S} 5$ & 482 & 506 & 884 & 939 & 883 & 909 & 978 & 1074 \\
\hline $\mathrm{S} 3 \rightarrow \mathrm{SW} 3$ & 615 & 941 & 882 & 941 & 914 & 942 & 978 & 1074 \\
\hline
\end{tabular}

ple fine-grained parameters that influence the performance, however, we do not model the values of those parameters in DNI. OMNeT++ uses for simulation a larger set of parameters than we are able to calculate in the transformation. The parameters that are not included in the DNI metamodel (e.g., TCP congestion algorithms, window size) are not transformed, and thus are set in $\mathrm{OMNeT}++$ to their default values. To increase the prediction accuracy, a simulator with finer granularity shall be used or the missing parameters should be provided manually.

Second, the calibration of the traffic patterns (here we use the ON-OFF traffic pattern) is done manually based on the protocol level traces. Manual calibration procedures are error prone and the errors usually cumulate when the network is under high load. In scenario 1, the main challenge was the extraction of the think time value. We depict schematically this phenomenon in Figure 6. Although we set the think time in the software as predefined value, it does not mean, that on the protocol level the respective value remains the same. In many simulators (also in $\mathrm{OMNeT}++$ ), the traffic generation process happens immediately, whereas in the software, the transmitted data must be copied between the respective memory cells and the respective mutexes need to be freed before a sleep instruction can be executed. The precise modeling of the generation delay may be omitted by infrequent data generations, however, by low think times this parameter has strong influence on the predicted values. The duration of the generation delay depends on the respective software implementation. In case of a single threaded implementation (e.g., in case of SBUS), the generation delay may take significant time, for example: for $1 \mathrm{MB}$ message and a $1 \mathrm{Gbit} / \mathrm{s}$ network interface, the generation of a message takes about $7 \mathrm{~ms}$. The precise calculation of the generation delay requires the available throughput to be known beforehand; this causes the fine model calibration challenging.

QPN. Both generated QPN models performed identically in low-load scenarios (1A-1D). The average prediction errors for the DNI QPN model are usually below $10 \%$ (with max-

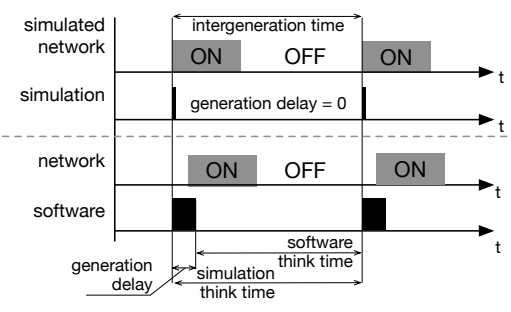

Figure 6: Differences between think times in simulation (upper part) and in the software (lower part).

imal error of $13.5 \%$ in scenario $1 \mathrm{~B}$ ), whereas for miniDNI QPN: $11.4 \%$ (maximum of $17.8 \%$ for scenario $1 \mathrm{E}$ ).

The differences between the two QPN models appear first when the network gets saturated. In scenario $1 \mathrm{E}$, the model generated from the miniDNI overestimates the measured throughput by about $160 \mathrm{Mbps}$ reporting higher throughput than achievable in the practice. The reason for that is the level of abstraction applied in the generated QPN modelthe protocol-related parameters are abstracted in the transformation. Additionally, the traffic calibration plays here an important role. Although the traffic patterns are not reflected in miniDNI, the DNI2miniDNI transformation relies on the traffic patterns to aggregate the traffic information. Thus, errors caused by the imprecise DNI calibration propagate to the QPN model.

\subsubsection{Simulation Time}

Along with the prediction precision, we evaluated the performance of the generated simulation models, i.e., the time needed to solve them. Depending on the situation, a less precise but quickly obtained result may be more valuable than precise but late predictions. During the execution of scenario 1 , we measured the execution time of the experiments and the three generated simulation models. We examined the run durations in two scenarios: $2 A$ and $2 B$. First $(2 A)$, we varied the traffic intensity for the prediction accuracy scenario. Second $(2 B)$, we increased the size of the network by adding servers, whereas the traffic intensity was constant.

The duration of simulations are measured using the time command on a non-virtualized server with Intel Xeon E31230 CPU, 16GB RAM, and Ubuntu Linux 12.04. We compile $\mathrm{OMNeT}++$ in the release mode (make $M O D E=$ release $)$ and exclude the TCL library $\left(N O_{-} T C L=1\right.$./configure). Simulations are run in the command line mode (cmdenv). Run durations for $\mathrm{OMNeT}++$ are estimated because of relatively long simulation times. First, we measure wall-clock time required to simulate 30 seconds of the respective real time (OMNeT parameter: sim-time-limit). During this simulation period, we observe the "simulation-seconds per second" parameter that describes the performance of the simulation. Then, we estimate the duration of the full simulation based on the real SBUS experiment duration and the measurements for 30 simulation-seconds. For selected simulation runs, we verify the estimations by simulating the complete experiment length. The verification shows, that the estimations are precise (up to $1 \%$ error).

Scenario 2A: Traffic Intensity. The simulation durations measured in scenario $2 \mathrm{~A}$ are presented in Table 4 and depicted in Figure 7a. Table 4 contains the durations of the original experiment for reference. The OMNeT ++ sim- 
Table 4: Scenario 2A: model solution duration (in seconds) for growing traffic intensity

\begin{tabular}{|c|r|r|r|r|r|}
\hline $\begin{array}{c}\text { Think } \\
\text { time }\end{array}$ & $\begin{array}{c}\text { SBUS } \\
\text { (real) }\end{array}$ & $\begin{array}{c}\text { OMNeT } \\
30 \mathrm{~s}\end{array}$ & $\begin{array}{c}\text { OMNeT } \\
\text { full }\end{array}$ & $\begin{array}{c}\text { QPN } \\
\text { DNI }\end{array}$ & $\begin{array}{c}\text { QPN } \\
\text { mDNI }\end{array}$ \\
\hline $100 \mathrm{~ms}$ & 1136 & 92 & 666 & 17 & 3 \\
\hline $50 \mathrm{~ms}$ & 670 & 175 & 3908 & 31 & 3 \\
\hline $35 \mathrm{~ms}$ & 528 & 234 & 4118 & 48 & 3 \\
\hline $20 \mathrm{~ms}$ & 416 & 351 & 4867 & 73 & 3 \\
\hline $10 \mathrm{~ms}$ & 348 & 519 & 6020 & 222 & 3 \\
\hline
\end{tabular}

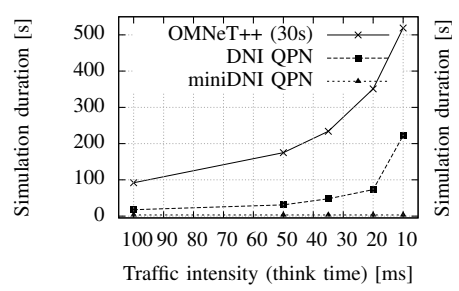

(a) Scenario $2 \mathrm{~A}$

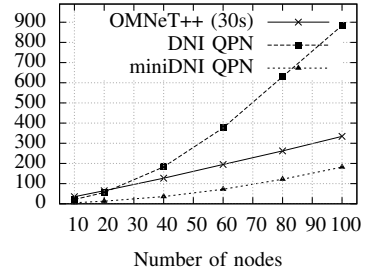

(b) Scenario $2 \mathrm{~B}$
Figure 7: Scenarios $2 A$ and $2 B$ : model solution duration (in seconds) for growing traffic intensity (2A) and network size $(2 \mathrm{~B})$

ulation models execute visibly slower than the QPNs - up to 100 minutes for $10 \mathrm{~ms}$ think time. We observe exponential growth of the simulation time for $\mathrm{OMNeT}++$ and $D N I$ QPN for growing traffic intensity. The miniDNI QPN model is insensitive to the traffic intensity and offers constant simulation time of 3 seconds. The exponential growth of simulation duration for OMNeT++ and DNI QPN is caused by the increasing number of events/tokens in the simulation model. The miniDNI QPN model abstracts the traffic patterns in the transformation and thus maintains constant number of tokens and constant simulation time.

Scenario 2B: Network Size. In this scenario, we assume a classical dumbbell topology with two directly connected switches and servers connected to them. We increase the number of servers connected to each switch while the traffic characteristics remain the same. Every node is set to transmit two $800 \mathrm{~KB}$ pictures per second. The experiment is over when each node has finished the transmission of 5000 pictures. We generate 6 setups containing $5,10,20,30,40$, and 50 nodes for each switch, i.e., 10, 20, 60, 80, and 100 nodes in total respectively. The simulation durations are presented in Table 5 and depicted in Figures $7 \mathrm{a}$ and $7 \mathrm{~b}$. Additionally, in the column „Transf.", we present the total time of the DNI model generation and the five model-to-model transformations. The source code of the transformations was not performance-optimized so the transformation duration can be further reduced.

In scenario $2 \mathrm{~B}$, we observe a linear growth of simulation time for OMNeT++ which is caused by the linear growth of the number of events in the simulation engine. This observation confirms the results obtained by Weingartner et al. in [35]. Similar dependency can be observed for miniDNI QPN where the number of tokens grows linearly with respect to the number of nodes. The DNI QPN model experiences exponential growth of the simulation duration. Despite the linear nature of the OMNeT++ run duration, the DNI QPN outperforms the full length run of $\mathrm{OMNeT}++$ by the factor
Table 5: Scenario 2B: transformation and model solution duration (in seconds) for growing network size

\begin{tabular}{|c|r|r|r|r|r|}
\hline Nodes & $\begin{array}{c}\text { OMNeT } \\
30 \text { s }\end{array}$ & $\begin{array}{c}\text { OMNeT } \\
\text { full }\end{array}$ & $\begin{array}{c}\text { QPN } \\
\text { DNI }\end{array}$ & $\begin{array}{c}\text { QPN } \\
\text { mDNI }\end{array}$ & Transf. \\
\hline $2 \times 5$ & 35 & 2953 & 21 & 5 & 25 \\
\hline $2 \times 10$ & 65 & 5484 & 56 & 13 & 67 \\
\hline $2 \times 20$ & 127 & 10716 & 183 & 36 & 149 \\
\hline $2 \times 30$ & 195 & 16453 & 376 & 72 & 277 \\
\hline $2 \times 40$ & 262 & 22106 & 630 & 122 & 446 \\
\hline $2 \times 50$ & 334 & 28181 & 884 & 182 & 692 \\
\hline
\end{tabular}

of 30. In scenario 2B, the miniDNI QPN model is solved on average 300 times faster than the respective full-length $\mathrm{OMNeT}++$ simulation; miniDNI QPN requires four times less simulation time than the respective DNI QPN model.

\section{CONCLUSIONS AND FUTURE WORK}

In this paper, we show that abstraction of selected details in network performance models can lead to only minor prediction accuracy degradation (up to $4 \%$ on average) but can accelerate the performance analysis by factor of 300 . We stress, that maximized accuracy of performance prediction requires highly-detailed, protocol-level modeling formalisms. In our approach, we accept lower prediction accuracy by providing technology independent generic modeling formalism. Despite the introduced abstractions in the DNI meta-model and fully automatic process of predictive model generation, we obtain good throughput prediction accuracy with maximal prediction error up to $18 \%$.

Using the DNI meta-model and the proposed model-tomodel transformations, we automatically obtain simulation models with different modeling granularity. The generated simulation models can be flexibly used according to the situation: less analysis overhead and less prediction accuracy or longer simulations but more accurate predictions. Our approach requires a single input DNI model and offers multiple predictive models without requiring any expertise in each of them. Non-experts can clearly benefit from our approach because they learn only one modeling formalism but receive multiple automatically generated models.

Obtaining good prediction accuracy requires careful model calibration. We formulate the technical challenges that need to be considered during the calibration process. For example, imprecise modeling of network traffic patterns, can visibly influence the predicted throughput values. To precisely tune the model parameters, a low-level trace-based calibration is recommended. We plan to support this process in our future work by providing tools and methods for automated or semi-automated calibration. Additionally, we plan to evaluate the DNI meta-model in SDN scenarios for data center networks. Furthermore, we plan to add support for additional performance metrics like transmission delay and network latency.

\section{REFERENCES}

[1] SDL combined with UML. ITU-T Z.109, 2000.

[2] uperf A network performance tool, 2012.

[3] OMG's Meta-ObjectFacility, 2014.

[4] J. Bacon, A. Beresford, D. Evans, D. Ingram, N. Trigoni, A. Guitton, and A. Skordylis. TIME: An open platform for capturing, processing and delivering transport-related data. 
In Proceedings of the 5th IEEE Consumer Communications and Networking Conference (CCNC), Las Vegas, 2008.

[5] S. Balsamo, A. di Marco, P. Inverardi, and M. Simeoni. Model-based performance prediction in software development: a survey. Software Engineering, IEEE Transactions on, 30(5):295-310, 2004.

[6] S. Becker, H. Koziolek, and R. Reussner. The Palladio component model for model-driven performance prediction. Journal of Systems and Software, 82(1):3-22, 2009.

[7] S. Bernardi and J. Merseguer. Performance evaluation of UML design with Stochastic Well-formed Nets. Journal of Systems and Software, 80(11):1843-1865, 2007.

[8] J. Britton and A. deVos. Cim-based standards and cim evolution. IEEE Transactions on Power Systems, 20(2):758-764, May 2005.

[9] F. Brosig, N. Huber, and S. Kounev. Architecture-Level Software Performance Abstractions for Online Performance Prediction. Elsevier Science of Computer Programming Journal (SciCo), 2013.

[10] V. Cortellessa, P. Pierini, R. Spalazzese, and A. Vianale. Moses: Modeling software and platform architecture in uml 2 for simulation-based performance analysis. In S. Becker, F. Plasil, and R. Reussner, editors, Quality of Software Architectures. Models and Architectures, volume 5281 of Lecture Notes in Computer Science, pages 86-102. Springer Berlin Heidelberg, 2008.

[11] N. de Wet and P. Kritzinger. Using UML models for the performance analysis of network systems. Comput. Netw., 49(5):627-642, 2005

[12] W. E. Denzel, J. Li, P. Walker, and Y. Jin. A framework for end-to-end simulation of high-performance computing systems. In Proceedings of the 1st International Conference on Simulation Tools and Techniques for Communications, Networks and Systems $\& 6$ Workshops, Simutools, pages 21:1-21:10, 2008 .

[13] I. Dietrich, F. Dressler, V. Schmitt, and R. German. SYNTONY: network protocol simulation based on standard-conform UML2 models. In Proc. of the Value Tools '0\%, pages 21:1-21:11, 2007.

[14] Q. Duan. Modeling and performance analysis on network virtualization for composite network-cloud service provisioning. In Services (SERVICES), 2011 IEEE World Congress on, pages 548-555, July 2011.

[15] A. J. Field, U. Harder, and P. G. Harrison. Network Traffic Behaviour in Switched Ethernet Systems. In MASCOTS 2002, 10th IEEE International Symposium on Modeling, Analysis, and Simulation of Computer and Telecommunications Systems, pages 32-42, October 2002.

[16] P. G. Harrison and N. M. Patel. Performance Modelling of Communication Networks and Computer Architectures. Addison-Wesley, 1993.

[17] N. Huber, M. von Quast, M. Hauck, and S. Kounev. Evaluating and Modeling Virtualization Performance Overhead for Cloud Environments. In Proc. of the 1st Int. Conf. on Cloud Computing and Services Science, pages 563-573, 2011.

[18] I. Kaj and J. Olsén. Throughput modeling and simulation for single connection tcp-tahoe. In J. M. de Souza, N. L. da Fonseca, and E. A. de Souza e Silva, editors, Teletraffic Engineering in the Internet Era, volume 4 of Teletraffic Science and Engineering, pages 705-718. Elsevier, 2001.

[19] D. Kolovos, R. Paige, and F. A. Polack. The Epsilon Transformation Language. In Theory and Practice of Model Transformations, vol. 5063 of LNCS, pages 46-60. Springer, 2008.

[20] S. Kounev, K. Bender, F. Brosig, N. Huber, and R. Okamoto. Automated Simulation-Based Capacity Planning for Enterprise Data Fabrics. In 4th International ICST Conference on Simulation Tools and Techniques, pages 27-36, 2011.

[21] L. Kristensen and K. Jensen. Specification and validation of an edge router discovery protocol for mobile ad hoc networks. In Integration of Software Specification Techniques for Applications in Engineering, pages 248-269. 2004.

[22] N. McKeown, T. Anderson, H. Balakrishnan, G. Parulkar, L. Peterson, J. Rexford, S. Shenker, and J. Turner. Openflow: enabling innovation in campus networks. SIGCOMM Comput. Commun. Rev., 38(2):69-74, 2008.

[23] Object Management Group (OMG). UML Profile for Modeling and Analysis of Real-Time and Embedded systems (MARTE), May 2006.

[24] A. Prakash, Z. Theisz, and R. Chaparadza. Formal methods for modeling, refining and verifying autonomic components of computer networks. In Transactions on Computational Science XV, pages 1-48. Springer, 2012.

[25] R. Puigjaner. Performance modelling of computer networks. In Proc. of the 2003 IFIP/ACM Latin America conf. on Towards a Latin American agenda for network research, LANC '03, pages 106-123, New York, NY, USA, 2003. ACM.

[26] G. Riley and T. Henderson. The ns-3 network simulator. In K. Wehrle, M. Günes, and J. Gross, editors, Modeling and Tools for Network Simulation, pages 15-34. Springer Berlin Heidelberg, 2010.

[27] P. Rygielski and S. Kounev. Data Center Network Throughput Analysis using Queueing Petri Nets. In 34th IEEE International Conference on Distributed Computing Systems Workshops. 4th International Workshop on Data Center Performance, (DCPerf 2014), pages 100-105, 2014.

[28] P. Rygielski and S. Kounev. Descartes Network Infrastructures (DNI) Manual: Meta-models, Transformations, Examples. Technical Report, 2014.

[29] P. Rygielski, S. Kounev, and S. Zschaler. Model-Based Throughput Prediction in Data Center Networks. In Proc. of the 2nd IEEE Int. Workshop on Measurements and Networking, pages 167-172, 2013.

[30] P. Rygielski, S. Zschaler, and S. Kounev. A metamodel for Performance Modeling of Dynamic Virtualized Network Infrastructures (Work-in-progess paper). In Proc. of the 4 th $A C M / S P E C$ Int. Conf. on Performance Engineering, pages 327-330. ACM, 2013.

[31] A. Varga. The OMNeT++ discrete event simulation system. In Proc. of the European Simulation Multi-conference, pages 319-324, 2001.

[32] J. G. von Kistowski, N. R. Herbst, and S. Kounev. LIMBO: A Tool For Modeling Variable Load Intensities. In Proceedings of the 5th ACM/SPEC International Conference on Performance Engineering (ICPE), pages 225-226. ACM, 2014.

[33] G. Wang and T. Ng. The impact of virtualization on network performance of amazon ec2 data center. In Proceedings of IEEE INFOCOM, pages 1-9, 2010.

[34] K. Wehrle, M. Günes, and J. Gross, editors. Modeling and Tools for Network Simulation. Springer, 2010.

[35] E. Weingartner, H. vom Lehn, and K. Wehrle. A performance comparison of recent network simulators. In IEEE International Conference on Communications, pages $1-5,2009$.

[36] M. Woodside, D. C. Petriu, D. B. Petriu, H. Shen, T. Israr, and J. Merseguer. Performance by unified model analysis (puma). In Proceedings of the 5th International Workshop on Software and Performance, pages 1-12. ACM, 2005.

[37] D. A. Zaitsev and T. R. Shmeleva. A Parametric Colored Petri Net Model of a Switched Network. Int. J. Communications, Network and System Sciences, (4):65-76, 2011. 